\title{
Walking and Other Common Physical Activities Among Adults with Arthritis - United States, 2019
}

\author{
Dana Guglielmo, $\mathrm{MPH}^{1,2}$; Louise B. Murphy, $\mathrm{PhD}^{1}$; Kristina A. Theis, $\mathrm{PhD}^{1}$; Michael A. Boring, MS ${ }^{1}$; Charles G. Helmick, MD ${ }^{1}$; \\ Kathleen B. Watson, $\mathrm{PhD}^{3}$; Lindsey M. Duca, $\mathrm{PhD}^{1,4}$; Erica L. Odom, DrPH ${ }^{1}$; Yong Liu, MD ${ }^{1}$; Janet B. Croft, $\mathrm{PhD}^{1}$
}

The numerous health benefits of physical activity include reduced risk for chronic disease and improved mental health and quality of life (1). Physical activity can improve physical function and reduce pain and fall risk among adults with arthritis, a group of approximately 100 conditions affecting joints and surrounding tissues (most commonly osteoarthritis, fibromyalgia, gout, rheumatoid arthritis, and lupus) (1). Despite these benefits, the 54.6 million U.S. adults currently living with arthritis are generally less active than adults without arthritis, and only $36.2 \%$ of adults with arthritis are aerobically active (i.e., meet aerobic physical activity guidelines*) (2). Little is known about which physical activities adults with arthritis engage in. CDC analyzed 2019 Behavioral Risk Factor Surveillance System (BRFSS) data to examine the most common nonwork-related physical activities among adults with arthritis who reported any physical activity during the past month, nationally and by state. In $2019,67.2 \%$ of adults with arthritis reported engaging in physical activity in the past month; among these persons, the most commonly reported activities were walking (70.8\%), gardening (13.3\%), and weightlifting (7.3\%). In 45 U.S. states, at least two thirds of adults with arthritis who engaged in physical activity reported walking. Health care providers can help inactive adults with arthritis become active and, by encouraging physical activity and referring these persons to evidence-based physical activity programs, improve their health and quality of life.

BRFSS is an ongoing, state-based landline and cellular telephone survey of noninstitutionalized U.S. adults aged $\geq 18$ years conducted by health departments in 50 states, the District of Columbia (DC), and U.S. territories. ${ }^{\dagger}$ In 2019, the median response rate among the 49 states included in this analysis $\$$ was $49.4 \%$ (range $=37.3 \%-73.1 \%$ ). ${ }^{9}$ Arthritis was defined as an affirmative response to the question, "Have you ever been told by a doctor or other health care professional that you have

\footnotetext{
* The earlier study used the 2008 Physical Activity Guidelines for Americans (https://health.gov/sites/default/files/2019-09/paguide.pdf), which are equivalent to the most recent (2018) Physical Activity Guidelines for Americans, 2nd Ed. https://health.gov/sites/default/files/2019-09/Physical_Activity_ Guidelines_2nd_edition.pdf

${ }^{\dagger}$ https://www.cdc.gov/brfss/about/index.htm

$\$$ This analysis included 49 states and the District of Columbia. In 2019, New Jersey did not collect enough data to meet the minimum requirement for inclusion in the BRFSS public-use data set.

https://www.cdc.gov/brfss/annual_data/2019/pdf/2019-sdqr-508.pdf
}

arthritis, rheumatoid arthritis, gout, lupus, or fibromyalgia?"** Engaging in physical activity was defined as responding "yes" to the question, "During the past month, other than your regular job, did you participate in any physical activities or exercises such as running, calisthenics, golf, gardening, or walking for exercise?" Among the 380,418 (92.8\%) BRFSS respondents in 49 states and DC who reported arthritis status, age, and physical activity status, 87,299 (22.9\%) reported having arthritis and engaging in physical activity. These participants were asked to report up to two activities in which they most frequently engaged from a list of 74 activities. ${ }^{\dagger \dagger}$

Unadjusted percentages for each activity were calculated for the combined 49 states and DC. Age-specific and ageadjusted ${ }^{\$}$ percentages for the three most commonly reported activities (walking, gardening, and weightlifting) were calculated for adults with arthritis engaging in nonwork-related physical activity by selected sociodemographic and healthrelated characteristics, including joint pain severity, body mass index, physical limitations, and self-rated health. Unadjusted state-specific prevalences of walking, gardening, and weightlifting among adults with arthritis were also estimated. Paired t-tests were performed to assess differences across subgroups for all variables, and linear trend tests using orthogonal linear contrasts were conducted for ordinal variables; all comparisons reported are statistically significant ( $\mathrm{p}$-value $<0.05$ ). Analyses accounted for BRFSS's complex sampling design, were weighted to be representative of each state, and were conducted using SAS (version 9.4; SAS Institute) and SUDAAN

\footnotetext{
** https://www.cdc.gov/arthritis/basics/types.html

$\dagger \dagger$ A specific activity was counted once if it was reported in response to one of the following questions: "What type of physical activity or exercise did you spend the most time doing during the past month?" or "What other type of physical activity gave you the next most exercise during the past month?" Participants who reported one activity but had missing data for the second most frequent activity (e.g., "don't know" or "refused") were included in the analysis. Among 87,299 adults with arthritis engaging in physical activity, 77,733 participants answered at least the first question (7,859 reported "Don't know," 366 refused, and 1,341 responses were missing). The 74 activities were organized into major headings using a modified version of the 2011 Compendium of Physical Activities by Ainsworth et al. (https://cdn-links. lww.com/permalink/mss/a/mss_43_8_2011_06_13_ainsworth_202093_ sdc1.pdf). Activities were grouped on the basis of similarity and on response rates, with activities having $<400$ respondents combined into "Other" categories corresponding to the major headings.

$\$ \$$ Age-adjusted estimates were generated in weighted logistic regression models that included age as a categorical covariate (18-44 years, 45-64 years, and $\geq 65$ years).
} 
(version 11.0; RTI International). This activity was reviewed by $\mathrm{CDC}$ and was conducted consistent with applicable federal law and CDC policy. 99

In $2019,67.2 \%$ of adults with arthritis engaged in nonwork-related physical activity in the past month; walking was the most commonly reported activity $(70.8 \%)$, followed by gardening (13.3\%), and weightlifting $(7.3 \%)$ (Table 1$)$. The percentage reporting walking was lowest among those 18-44 years (63.7\%) (Table 2). The age-adjusted prevalence of walking was higher among women $(76.0 \%)$ than among men (63.9\%), higher among non-Hispanic Black (75.4\%) adults than among non-Hispanic White $(70.0 \%)$ and non-Hispanic other/multiple race adults (68.3\%), and higher among those who were unable to work or disabled $(79.0 \%)$ compared with those adults with other employment statuses $(67.7 \%-74.8 \%)$. The age-adjusted percentage of adults with arthritis who reported walking increased with increasing joint pain severity

9945 C.F.R. part 46.102(1) (2), 21 C.F.R. part 56; 42 U.S.C. Sect. 241(d); 5 U.S.C. Sect. 552a; 44 U.S.C. Sect. 3501 et seq.

TABLE 1. Weighted unadjusted percentages of adults with arthritis* who reported engaging in physical activity in the past month, ${ }^{\dagger}$ reporting first or second most frequent activities ${ }^{\S}$ - Behavioral Risk Factor Surveillance System, United States, 2019

\begin{tabular}{|c|c|c|}
\hline Activity group** & No. of respondents & $\%(95 \% \mathrm{Cl})$ \\
\hline Walking or backpacking & 62,902 & $72.1(71.4-72.7)$ \\
\hline Walking & 61,931 & $70.8(70.2-71.4)$ \\
\hline Hiking or backpacking & 1,312 & $1.6(1.5-1.8)$ \\
\hline Lawn and garden & 18,297 & $19.6(19.1-20.2)$ \\
\hline Gardening & 12,094 & $13.3(12.8-13.8)$ \\
\hline Yard work & 6,585 & $6.6(6.3-7.0)$ \\
\hline Muscle strengthening & 9,885 & $12.8(12.3-13.2)$ \\
\hline Weightlifting & 5,357 & $7.3(7.0-7.7)$ \\
\hline Calisthenics $^{\dagger \dagger}$ & 2,014 & $2.6(2.4-2.8)$ \\
\hline Yoga & 2,368 & $2.7(2.5-2.9)$ \\
\hline Pilates & 349 & $0.4(0.3-0.5)$ \\
\hline $\begin{array}{l}\text { Aerobic conditioning } \\
\text { exercise }\end{array}$ & 9,196 & $10.0(9.6-10.4)$ \\
\hline Bicycling machine exercise & 4,241 & $4.5(4.2-4.8)$ \\
\hline Aerobics video or class & 2,210 & $2.4(2.2-2.6)$ \\
\hline $\begin{array}{l}\text { Elliptical or elliptical fitness } \\
\text { crosstrainer machine } \\
\text { exercise }\end{array}$ & 1,675 & $2.1(1.9-2.3)$ \\
\hline $\begin{array}{l}\text { Stair climbing or } \\
\text { StairMaster }\end{array}$ & 959 & $0.9(0.8-1.1)$ \\
\hline $\begin{array}{l}\text { Other aerobic conditioning } \\
\text { exercise }\end{array}$ & 377 & $0.4(0.4-0.5)$ \\
\hline Home activities ${ }^{\S \S}$ & 7,621 & $7.9(7.5-8.2)$ \\
\hline Sports & 5,115 & $6.3(6.0-6.7)$ \\
\hline Golf & 2,571 & $2.9(2.7-3.1)$ \\
\hline Bowling & 394 & $0.5(0.4-0.6)$ \\
\hline Tennis & 379 & $0.5(0.4-0.6)$ \\
\hline Other sports & 1,881 & $2.6(2.4-2.9)$ \\
\hline Running or jogging & 2,459 & $4.5(4.2-4.9)$ \\
\hline Water activities & 3,654 & $4.4(4.2-4.7)$ \\
\hline Swimming & 3,345 & $4.1(3.8-4.4)$ \\
\hline Other water activities & 315 & $0.3(0.3-0.4)$ \\
\hline
\end{tabular}

and body mass index, and decreased with increasing education, income, and self-rated health.

The percentage of adults with arthritis who reported gardening increased with age from $7.0 \%$ among adults aged $18-44$ years to $16.4 \%$ among those aged $\geq 65$ years. The ageadjusted prevalence of gardening was higher among women (15.1\%) than among men (10.9\%), and higher among nonHispanic White adults (14.4\%) than among non-Hispanic American Indian/Alaska Native adults (8.0\%) and non-Hispanic Black adults (7.8\%). The percentage reporting gardening was lower among those without a high school diploma (10.5\%) than among persons with higher levels of educational attainment $(12.7 \%-14.5 \%)$. Gardening prevalence increased with increasing rurality.

TABLE 1. (Continued) Weighted unadjusted percentages of adults with arthritis* who reported engaging in physical activity in the past month, ${ }^{\dagger}$ reporting first or second most frequent activities ${ }^{\S}$ Behavioral Risk Factor Surveillance System, United States, 2019

\begin{tabular}{lcc}
\hline Activity group $^{* *}$ & No. of respondents & $\%(95 \% \mathrm{Cl})$ \\
\hline Bicycling & 3,314 & $4.3(4.0-4.6)$ \\
Dancing & 966 & $1.3(1.2-1.5)$ \\
Fishing and hunting & 716 & $0.9(0.8-1.0)$ \\
Farm or ranch work & 1,182 & $0.9(0.8-1.0)$ \\
Winter activities & 900 & $0.6(0.5-0.7)$ \\
Snow shoveling by hand & 626 & $0.4(0.4-0.5)$ \\
Other winter activities & 286 & $0.2(0.1-0.2)$ \\
\hline
\end{tabular}

Abbreviations: BRFSS = Behavioral Risk Factor Surveillance System; $\mathrm{Cl}=$ confidence interval.

* Respondents were classified as having arthritis if they responded "Yes" to the question, "Have you ever been told you have some form of arthritis, rheumatoid arthritis, gout, lupus, or fibromyalgia?"

${ }^{\dagger}$ Respondents with arthritis were classified as engaging in physical activity if they responded "Yes" to the question, "During the past month, other than your regular job, did you participate in any physical activities or exercises such as running, calisthenics, golf, gardening, or walking for exercise?"

$\S$ Those who engaged in physical activity were classified as participating in an activity if they reported this activity in response to two questions: 1) "What type of physical activity or exercise did you spend the most time doing during the past month?" or 2) "What other type of physical activity gave you the next most exercise during the past month?" Participants who reported one activity but had missing data for the other most frequent activity (e.g., "don't know" or "refused") were included in the analysis. The sum of respondents for all activities exceeds the total number of respondents since each respondent could report up to two activities. Survey interviewers coded activities not listed among the 74 activities in the BRFSS Activity List for Common Leisure Activities into a single, heterogeneous "other" category representing a wide variety of different activities ( $n=13,241 ; 13.7 \%$ [95\% Cl: 13.2-14.1]).

"In 2019, New Jersey did not collect enough data to meet the minimum requirement for inclusion in the BRFSS public-use data set.

** The 74 activities were organized into major headings using a modified version of the 2011 Compendium of Physical Activities by Ainsworth, et. al. (https:// cdn-links.Iww.com/permalink/mss/a/mss_43_8_2011_06_13_ ainsworth_202093_sdc1.pdf). Activities were grouped on the basis of similarity and on response rates, with activities having $<400$ respondents combined into "Other" categories corresponding to the major headings.

t+ Some calisthenics activities might be classified as aerobic conditioning exercise.

$\S \S$ Home activities included household activities (e.g., vacuuming, dusting, or home repair), child care, carpentry, and painting or wallpapering. 
TABLE 2. Age-specific and age-adjusted* percentages of reporting walking, gardening, or weightlifting as a first or second most frequent activity $^{\dagger}$ among adults with arthritis ${ }^{\S}$ who reported engaging in physical activity in the past month," by selected characteristics — Behavioral Risk Factor Surveillance System, United States, ${ }^{* *} 2019$

\begin{tabular}{|c|c|c|c|c|}
\hline \multirow[b]{2}{*}{ Characteristic } & \multirow{2}{*}{$\begin{array}{l}\text { No. of adults with arthritis } \\
\text { engaging in physical activity }\end{array}$} & \multicolumn{3}{|c|}{ Age-adjusted \% $(95 \% \mathrm{Cl})^{*}$} \\
\hline & & Walking & Gardening & Weightlifting \\
\hline Overall & 87,299 & $70.0(69.3-70.7)$ & $10.7(10.3-11.2)$ & $10.3(9.8-10.9)$ \\
\hline \multicolumn{5}{|l|}{ Sociodemographic characteristic } \\
\hline \multicolumn{5}{|l|}{ Age group, yrs (unadjusted) } \\
\hline $18-44$ & 8,107 & $63.7(61.8-65.5)$ & $7.0(6.1-8.0)$ & $12.3(11.1-13.6)$ \\
\hline $45-64$ & 30,635 & $73.5(72.6-74.5)$ & $12.8(12.0-13.7)$ & $7.0(6.5-7.6)$ \\
\hline$\geq 65$ & 48,557 & $71.2(70.3-72.0)$ & $16.4(15.7-17.1)$ & $5.5(5.1-6.0)$ \\
\hline \multicolumn{5}{|l|}{ Sex } \\
\hline Male & 34,886 & $63.9(62.9-64.9)$ & $10.9(10.2-11.6)$ & $10.9(10.2-11.5)$ \\
\hline Female & 52,413 & $76.0(75.2-76.7)$ & $15.1(14.5-15.8)$ & $4.7(4.3-5.1)$ \\
\hline \multicolumn{5}{|l|}{ Race/Ethnicity } \\
\hline White, non-Hispanic & 72,415 & $70.0(69.4-70.7)$ & $14.4(13.9-14.9)$ & $7.3(6.9-7.7)$ \\
\hline Black, non-Hispanic & 5,607 & $75.4(73.3-77.4)$ & $7.8(6.7-9.1)$ & $7.9(6.6-9.4)$ \\
\hline Hispanic & 3,059 & $72.8(69.7-75.7)$ & $11.7(8.9-15.2)$ & $7.3(5.8-9.2)$ \\
\hline Asian, non-Hispanic & 794 & $72.1(65.2-78.0)$ & $11.4(7.4-17.1)$ & $8.7(5.7-13.1)$ \\
\hline American Indian or Alaska Native, non-Hispanic & 1,290 & $74.8(68.6-80.2)$ & $8.0(5.4-11.8)$ & $4.4(3.1-6.3)$ \\
\hline Other/Multiple race, non-Hispanic & 2,495 & $68.3(64.5-71.9)$ & $14.3(11.7-17.3)$ & $6.0(4.6-7.9)$ \\
\hline \multicolumn{5}{|l|}{ Highest level of education } \\
\hline Less than high school graduate & 4,963 & $76.7(74.5-78.7)$ & $10.5(9.1-12.0)$ & $3.2(2.4-4.3)$ \\
\hline High school graduate or equivalent & 21,782 & $71.7(70.4-72.8)$ & $13.6(12.6-14.6)$ & $5.5(4.9-6.2)$ \\
\hline Technical school or some college & 26,276 & $70.8(69.6-71.9)$ & $14.5(13.7-15.4)$ & $6.7(6.1-7.4)$ \\
\hline College degree or higher & 34,120 & $68.1(67.1-69.1)$ & $12.7(12.0-13.5)$ & $11.2(10.5-11.9)$ \\
\hline \multicolumn{5}{|l|}{ Employment status } \\
\hline Employed or self-employed & 30,192 & $67.7(66.6-68.8)$ & $13.0(12.1-13.9)$ & $9.2(8.6-9.9)$ \\
\hline Unemployed & 2,822 & $74.8(71.2-78.1)$ & $11.6(9.5-14.1)$ & $5.8(4.2-8.1)$ \\
\hline Retired & 41,668 & $71.0(69.8-72.2)$ & $14.2(13.3-15.1)$ & $6.7(6.0-7.6)$ \\
\hline Unable to work or disabled & 8,058 & $79.0(77.1-80.7)$ & $11.1(9.9-12.5)$ & $2.1(1.7-2.7)$ \\
\hline Student or homemaker & 4,206 & $73.5(70.8-76.0)$ & $14.6(12.7-16.7)$ & $7.1(5.6-9.1)$ \\
\hline \multicolumn{5}{|l|}{ Federal poverty level ${ }^{\dagger \dagger}$} \\
\hline$\leq 125 \% \mathrm{FPL}$ & 11,478 & $77.3(75.7-78.8)$ & $11.0(10.0-12.2)$ & $3.4(2.8-4.1)$ \\
\hline$>125 \%$ to $\leq 200 \% \mathrm{FPL}$ & 12,531 & $72.8(71.2-74.3)$ & $13.4(12.2-14.7)$ & $5.5(4.6-6.4)$ \\
\hline$>200 \%$ to $\leq 400 \% \mathrm{FPL}$ & 21,874 & $70.7(69.4-71.9)$ & $14.7(13.8-15.7)$ & $7.2(6.5-7.9)$ \\
\hline$>400 \% \mathrm{FPL}$ & 26,569 & $66.7(65.5-67.8)$ & $13.3(12.4-14.2)$ & $11.2(10.4-12.0)$ \\
\hline \multicolumn{5}{|l|}{ Sexual orientation ${ }^{\S \S}$} \\
\hline Straight & 48,499 & $70.6(69.7-71.4)$ & $13.9(13.3-14.6)$ & $7.0(6.5-7.5)$ \\
\hline Lesbian, gay, bisexual, queer, or questioning & 2,700 & $74.0(70.9-76.9)$ & $12.1(9.9-14.8)$ & $6.6(4.9-8.8)$ \\
\hline \multicolumn{5}{|l|}{ Urban-rural status } \\
\hline Large central metro & 11,279 & $72.4(70.8-73.9)$ & $11.8(10.6-13.2)$ & $8.5(7.6-9.4)$ \\
\hline Large fringe metro & 15,941 & $67.9(66.6-69.2)$ & $12.9(12.1-13.8)$ & $8.2(7.4-9.1)$ \\
\hline Medium metro & 18,392 & $70.3(69.1-71.4)$ & $13.4(12.6-14.3)$ & $7.0(6.4-7.6)$ \\
\hline Small metro & 12,587 & $70.2(68.7-71.7)$ & $13.9(12.8-15.1)$ & $6.8(6.0-7.7)$ \\
\hline Micropolitan & 14,468 & $69.6(68.2-71.1)$ & $14.5(13.5-15.6)$ & $5.6(4.9-6.5)$ \\
\hline Noncore & 14,632 & $71.9(70.3-73.5)$ & $15.7(14.4-17.0)$ & $4.0(3.3-4.7)$ \\
\hline \multicolumn{5}{|l|}{ Health-related characteristic } \\
\hline \multicolumn{5}{|l|}{ Joint pain severity*** } \\
\hline None/Mild & 46,371 & $69.1(68.2-70.0)$ & $13.5(12.8-14.2)$ & $9.4(8.8-10.0)$ \\
\hline Moderate & 20,280 & $71.6(70.3-72.8)$ & $13.5(12.6-14.4)$ & $6.5(5.8-7.3)$ \\
\hline Severe & 19,421 & $73.6(72.4-74.9)$ & $12.7(11.8-13.7)$ & $4.3(3.7-4.9)$ \\
\hline \multicolumn{5}{|l|}{ Body mass index $\left(\mathrm{kg} / \mathrm{m}^{2}\right)$} \\
\hline Underweight or healthy weight $(<25)$ & 22,816 & $68.5(67.2-69.7)$ & $13.5(12.6-14.5)$ & $7.9(7.2-8.7)$ \\
\hline Overweight $(25$ to $<30)$ & 30,115 & $69.1(68.0-70.1)$ & $13.7(12.8-14.6)$ & $8.9(8.3-9.7)$ \\
\hline Obese $(\geq 30)$ & 30,171 & $73.6(72.6-74.5)$ & $12.9(12.1-13.6)$ & $5.9(5.3-6.4)$ \\
\hline \multicolumn{5}{|l|}{ Mobility limitations ${ }^{\dagger+\dagger}$} \\
\hline No & 63,303 & $69.7(68.9-70.4)$ & $13.9(13.3-14.5)$ & $8.6(8.1-9.0)$ \\
\hline Yes & 23,530 & $73.9(72.8-75.1)$ & $11.8(10.9-12.7)$ & $3.9(3.3-4.4)$ \\
\hline \multicolumn{5}{|l|}{ Arthritis-attributable activity limitations $\mathrm{s}^{\S \S \S}$} \\
\hline No & 54,910 & $70.1(69.3-70.9)$ & $13.3(12.7-13.9)$ & $8.6(8.1-9.1)$ \\
\hline Yes & 31,562 & $71.9(70.9-72.9)$ & $13.4(12.6-14.1)$ & $5.3(4.9-5.8)$ \\
\hline
\end{tabular}

See table footnotes on the next page. 
TABLE 2. (Continued) Age-specific and age-adjusted* percentages of reporting walking, gardening, or weightlifting as a first or second most frequent activity ${ }^{\dagger}$ among adults with arthritis ${ }^{\S}$ who reported engaging in physical activity in the past month, ${ }^{\mathfrak{\Upsilon}}$ by selected characteristics Behavioral Risk Factor Surveillance System, United States, ${ }^{* *} 2019$

\begin{tabular}{|c|c|c|c|c|}
\hline \multirow[b]{2}{*}{ Characteristic } & \multirow{2}{*}{$\begin{array}{l}\text { No. of adults with arthritis } \\
\text { engaging in physical activity }\end{array}$} & \multicolumn{3}{|c|}{ Age-adjusted $\%(95 \% \mathrm{Cl})^{*}$} \\
\hline & & Walking & Gardening & Weightlifting \\
\hline \multicolumn{5}{|c|}{ Arthritis-attributable work limitations } \\
\hline No & 63,083 & $70.1(69.3-70.8)$ & $13.0(12.5-13.6)$ & $8.7(8.3-9.3)$ \\
\hline Yes & 22,660 & $72.4(71.3-73.6)$ & $14.0(13.1-15.0)$ & $4.5(4.0-5.0)$ \\
\hline \multicolumn{5}{|l|}{ Self-rated health } \\
\hline Excellent or very good & 35,055 & $67.5(66.4-68.4)$ & $13.2(12.5-14.0)$ & $10.5(9.8-11.2)$ \\
\hline Good & 31,206 & $72.1(71.1-73.1)$ & $14.5(13.6-15.4)$ & $6.2(5.7-6.8)$ \\
\hline Fair or poor & 20,858 & $74.1(72.9-75.3)$ & $11.8(11.0-12.7)$ & $4.2(3.6-4.8)$ \\
\hline
\end{tabular}

Abbreviations: $\mathrm{Cl}$ = confidence interval; $\mathrm{FPL}=$ federal poverty level.

* Except for age groups, age-adjusted estimates were generated in weighted logistic regression models that included age as a categorical covariate (18-44 years, $45-64$ years, and $\geq 65$ years).

† Those who were engaging in physical activity were classified as participating in an activity if they reported this activity for one of two questions: 1) "What type of physical activity or exercise did you spend the most time doing during the past month?" or 2) "What other type of physical activity gave you the next most exercise during the past month?" Participants who reported one activity but had missing data for the second most frequent activity (e.g., "don't know" or "refused") were included in the analysis.

$\S$ Respondents were classified as having arthritis if they responded "yes" to the question, "Have you ever been told by a doctor or other health care professional that you have arthritis, rheumatoid arthritis, gout, lupus, or fibromyalgia?"

" Respondents with arthritis were classified as engaging in physical activity if they responded "yes" to the question, "During the past month, other than your regular job, did you participate in any physical activities or exercises such as running, calisthenics, golf, gardening, or walking for exercise?"

** In 2019, New Jersey did not collect enough data to meet the minimum requirement for inclusion in the BRFSS public-use data set.

${ }^{t+}$ FPL is the ratio of total family income to federal poverty level per family size. Overall, 14,847 adults with arthritis engaging in physical activity had missing FPL data.

$\S \S$ Sexual orientation was asked in 30 states (Alaska, Arizona, Colorado, Connecticut, Delaware, Florida, Georgia, Hawaii, Idaho, lowa, Kansas, Louisiana, Maryland, Minnesota, Mississippi, Montana, New York, North Carolina, Ohio, Oklahoma, Rhode Island, South Carolina, Tennessee, Texas, Utah, Vermont, Virginia, Washington, West Virginia, and Wisconsin). A total of 788 adults with arthritis who engaged in physical activity refused to answer.

१ๆ Urban-rural status was categorized using the National Center for Health Statistics 2013 Urban-Rural Classification Scheme for Counties. https://www.cdc.gov/ nchs/data/series/sr_02/sr02_166.pdf

*** For the question, "On a scale of 0 to 10 where 0 is no pain or aching and 10 is pain or aching as bad as it can be, during the past 30 days, how bad was your joint pain on average," an answer of 0-4 was defined as none/mild, an answer of 5-6 was defined as moderate, and an answer of 7-10 was defined as severe.

$t_{+\dagger}$ Respondents were classified as having mobility limitations if they responded "yes" to the question, "Do you have serious difficulty walking or climbing stairs?"

$\S \S \S$ Respondents were classified as having arthritis-attributable activity limitations if they responded "yes" to the question, "Are you now limited in any way in any of your usual activities because of arthritis or joint symptoms?"

กศๆ Respondents were classified as having arthritis-attributable work limitations if they responded "yes" to the question, "In this next question, we are referring to work for pay. Do arthritis or joint symptoms now affect whether you work, the type of work you do, or the amount of work you do?"

The prevalence of weightlifting was highest among those aged $18-44$ years $(12.3 \%)$, declined with age, and was higher among men $(10.9 \%)$ than among women $(4.7 \%)$ and higher among those who were employed or self-employed (9.2\%) than among those who were unable to work or disabled $(2.1 \%)$. Weightlifting prevalence increased with increasing education, income, and self-rated health and decreased with increasing joint pain severity and rurality.

The median state-specific unadjusted percentage of adults with arthritis who reported walking was $70.5 \%$ (range $=62.9 \%$ [Hawaii] to 75.4\% [Alabama]) (Table 3). The median percentage who reported gardening was $12.6 \%$ (range $=3.8 \%[\mathrm{DC}]$ to $17.6 \%$ [Florida], and the median who reported weightlifting was $7.1 \%$ (range $=3.6 \%$ [Maine] to $13.9 \%$ [DC]).

\section{Discussion}

In 2019, walking was overwhelmingly the most common activity among adults with arthritis who engaged in nonwork-related physical activity in the past month, followed by gardening and weightlifting. The most common activities in this report parallel the activities for adults with mobility disabilities, whose most common activities in 2017 were walking and gardening (3). These similarities are expected because arthritis is a leading cause of disability (4). Despite arthritis being a cause of pain and disability, walking prevalence increased with increasing joint pain severity. A previous report on walking using national data described a similar finding, specifically for lower extremity joint pain (5). Collectively, these findings might signify that the presence of pain might not automatically preclude walking, other physical activities, and their associated benefits.

Walking is an ideal activity for adults with arthritis because it can be inexpensive, safe, convenient, low-impact, and adaptable to individual fitness levels.*** The American College of Rheumatology and the Arthritis Foundation recommend that health care providers offer specific guidance to patients with arthritis regarding physical activity ( () . This report identifies activities to which adults with arthritis seem amenable. These

\footnotetext{
*** https://www.hhs.gov/sites/default/files/call-to-action-walking-and-walkablecommunites.pdf
} 
TABLE 3. Unadjusted reported prevalence of walking, gardening, or weightlifting as a first or second most frequent activity* among adults with arthritis $^{\dagger}$ who reported engaging in physical activity in the past month ${ }^{\S}$ - Behavioral Risk Factor Surveillance System, United States, ${ }^{\natural} 2019$

\begin{tabular}{|c|c|c|c|c|c|c|}
\hline \multirow[b]{2}{*}{ Jurisdiction } & \multicolumn{2}{|c|}{ Walking } & \multicolumn{2}{|c|}{ Gardening } & \multicolumn{2}{|c|}{ Weightlifting } \\
\hline & Weighted no.** & $\begin{array}{l}\text { Unadjusted } \\
\%(95 \% \mathrm{CI})\end{array}$ & Weighted no.** & $\begin{array}{l}\text { Unadjusted } \\
\%(95 \% \mathrm{Cl})\end{array}$ & Weighted no.** & $\begin{array}{l}\text { Unadjusted } \\
\%(95 \% \mathrm{CI})\end{array}$ \\
\hline Alabama & 548,000 & $75.4(72.6-78.0)$ & 111,000 & $15.3(13.3-17.6)$ & 39,000 & $5.3(4.0-7.1)$ \\
\hline Alaska & 60,000 & 74.8 (69.7-79.3) & 7,000 & $9.0(6.2-12.9)$ & 5,000 & $5.9(4.0-8.8)$ \\
\hline Arizona & 603,000 & $73.3(69.9-76.4)$ & 82,000 & $9.9(8.2-12.0)$ & 73,000 & $8.9(6.9-11.5)$ \\
\hline Arkansas & 278,000 & $71.6(67.8-75.0)$ & 62,000 & $16.0(13.6-18.8)$ & 19,000 & $5.0(3.4-7.2)$ \\
\hline California & $3,053,000$ & $74.2(71.3-76.8)$ & 653,000 & $15.9(13.8-18.2)$ & 330,000 & $8.0(6.6-9.8)$ \\
\hline Colorado & 489,000 & $67.7(65.1-70.3)$ & 59,000 & $8.2(6.8-9.8)$ & 80,000 & $11.0(9.4-12.9)$ \\
\hline Connecticut & 300,000 & $70.2(67.3-72.9)$ & 55,000 & $13.0(11.2-15.0)$ & 30,000 & $6.9(5.5-8.7)$ \\
\hline Delaware & 86,000 & $70.4(65.4-74.9)$ & 16,000 & $13.0(10.3-16.3)$ & 7,000 & $5.3(3.9-7.4)$ \\
\hline District of Columbia & 42,000 & $70.5(64.8-75.6)$ & 2,000 & $3.8(2.3-6.2)$ & 8,000 & $13.9(9.7-19.7)$ \\
\hline Florida & $1,867,000$ & $68.9(65.7-72.0)$ & 477,000 & $17.6(14.5-21.2)$ & 182,000 & $6.7(5.2-8.7)$ \\
\hline Georgia & 793,000 & $70.2(66.2-73.9)$ & 137,000 & $12.2(9.8-15.0)$ & 96,000 & $8.5(5.9-12.1)$ \\
\hline Hawaii & 100,000 & $62.9(59.3-66.4)$ & 24,000 & $15.3(13.0-17.9)$ & 12,000 & $7.3(5.5-9.5)$ \\
\hline Idaho & 141,000 & $63.3(58.4-67.9)$ & 37,000 & $16.7(13.7-20.2)$ & 13,000 & $5.8(3.8-8.7)$ \\
\hline Illinois & $1,067,000$ & $67.6(64.2-70.9)$ & 209,000 & $13.2(11.1-15.7)$ & 130,000 & $8.3(6.5-10.5)$ \\
\hline Indiana & 562,000 & $73.0(70.2-75.6)$ & 80,000 & $10.4(8.7-12.3)$ & 55,000 & $7.1(5.6-8.9)$ \\
\hline lowa & 276,000 & $68.9(66.4-71.2)$ & 46,000 & $11.5(10.0-13.1)$ & 27,000 & $6.8(5.6-8.3)$ \\
\hline Kansas & 257,000 & $73.3(70.9-75.5)$ & 43,000 & $12.4(10.9-14.1)$ & 25,000 & $7.2(5.8-8.8)$ \\
\hline Kentucky & 460,000 & $71.8(68.4-75.0)$ & 89,000 & $13.8(11.7-16.3)$ & 38,000 & $5.9(4.3-8.2)$ \\
\hline Louisiana & 399,000 & $72.5(68.6-76.1)$ & 88,000 & $15.9(13.3-19.0)$ & 35,000 & $6.4(4.4-9.3)$ \\
\hline Maine & 141,000 & $68.4(65.5-71.1)$ & 33,000 & $15.9(13.9-18.0)$ & 7,000 & $3.6(2.5-5.2)$ \\
\hline Maryland & 522,000 & 71.5 (69.4-73.6) & 81,000 & $11.1(9.9-12.5)$ & 62,000 & $8.6(7.2-10.1)$ \\
\hline Massachusetts & 593,000 & $68.6(65.3-71.7)$ & 109,000 & $12.6(10.5-15.0)$ & 52,000 & $6.0(4.6-7.7)$ \\
\hline Michigan & $1,132,000$ & $73.4(71.0-75.6)$ & 152,000 & $9.8(8.4-11.4)$ & 111,000 & $7.2(5.9-8.7)$ \\
\hline Minnesota & 469,000 & $71.1(69.0-73.0)$ & 103,000 & $15.6(14.1-17.2)$ & 40,000 & $6.1(5.1-7.3)$ \\
\hline Mississippi & 243,000 & 73.7 (69.4-77.6) & 43,000 & $12.9(10.5-15.7)$ & 18,000 & $5.6(3.9-7.8)$ \\
\hline Missouri & 527,000 & $67.4(64.0-70.6)$ & 69,000 & $8.8(7.1-10.9)$ & 43,000 & $5.5(4.3-7.1)$ \\
\hline Montana & 119,000 & $68.2(65.4-71.0)$ & 22,000 & $12.6(10.8-14.8)$ & 14,000 & $8.2(6.6-10.1)$ \\
\hline Nebraska & 155,000 & $72.6(70.3-74.8)$ & 23,000 & $10.9(9.5-12.5)$ & 15,000 & $7.2(5.8-8.8)$ \\
\hline Nevada & 251,000 & $68.8(62.0-74.8)$ & 27,000 & $7.5(5.3-10.7)$ & 36,000 & $9.8(6.3-14.8)$ \\
\hline New Hampshire & 136,000 & $71.6(68.2-74.7)$ & 24,000 & $12.5(10.5-14.8)$ & 12,000 & $6.2(4.5-8.3)$ \\
\hline New Mexico & 204,000 & $73.6(70.2-76.7)$ & 29,000 & $10.5(8.6-12.8)$ & 26,000 & $9.2(7.3-11.6)$ \\
\hline New York & $1,509,000$ & $73.5(70.9-76.0)$ & 202,000 & 9.8 (8.4-11.5) & 148,000 & $7.2(5.8-8.9)$ \\
\hline North Carolina & 970,000 & $69.0(65.1-72.7)$ & 242,000 & $17.2(14.4-20.5)$ & 97,000 & $6.9(5.2-9.0)$ \\
\hline North Dakota & 64,000 & $65.1(61.1-68.9)$ & 10,000 & $10.2(8.2-12.5)$ & 9,000 & $9.6(7.4-12.4)$ \\
\hline Ohio & $1,123,000$ & $68.8(66.3-71.3)$ & 177,000 & $10.8(9.6-12.3)$ & 107,000 & $6.5(5.2-8.2)$ \\
\hline Oklahoma & 325,000 & $71.1(67.6-74.3)$ & 42,000 & $9.1(7.5-11.1)$ & 35,000 & $7.6(5.8-9.9)$ \\
\hline Oregon & 398,000 & $65.5(62.1-68.8)$ & 102,000 & $16.8(14.3-19.8)$ & 40,000 & $6.6(4.9-8.8)$ \\
\hline Pennsylvania & $1,277,000$ & $67.2(64.0-70.3)$ & 241,000 & $12.7(10.7-14.9)$ & 164,000 & $8.6(6.8-10.9)$ \\
\hline Rhode Island & 101,000 & $71.2(67.8-74.4)$ & 19,000 & $13.3(11.2-15.7)$ & 10,000 & $7.0(5.2-9.4)$ \\
\hline South Carolina & 504,000 & $75.4(72.6-78.0)$ & 99,000 & $14.8(12.7-17.3)$ & 48,000 & $7.2(5.6-9.1)$ \\
\hline South Dakota & 68,000 & $65.3(59.1-71.0)$ & 8,000 & $8.1(5.7-11.3)$ & 9,000 & $8.8(6.0-12.6)$ \\
\hline Tennessee & 662,000 & $74.3(71.0-77.3)$ & 117,000 & $13.1(11.0-15.7)$ & 64,000 & $7.1(5.4-9.5)$ \\
\hline Texas & $1,880,000$ & $70.2(66.4-73.7)$ & 386,000 & $14.4(11.9-17.3)$ & 215,000 & $8.0(6.1-10.6)$ \\
\hline Utah & 264,000 & $67.4(65.1-69.7)$ & 42,000 & $10.8(9.4-12.4)$ & 36,000 & $9.3(7.9-10.8)$ \\
\hline Vermont & 64,000 & $72.0(68.8-75.1)$ & 11,000 & $12.8(10.8-15.0)$ & 5,000 & $5.5(3.9-7.5)$ \\
\hline Virginia & 765,000 & $70.0(67.3-72.7)$ & 130,000 & $11.9(10.2-13.8)$ & 68,000 & $6.3(5.1-7.7)$ \\
\hline Washington & 739,000 & $71.1(68.9-73.2)$ & 177,000 & $17.1(15.4-18.9)$ & 65,000 & $6.2(5.3-7.4)$ \\
\hline West Virginia & 237,000 & $68.4(65.3-71.3)$ & 35,000 & $10.1(8.3-12.2)$ & 18,000 & $5.3(4.0-7.0)$ \\
\hline Wisconsin & 605,000 & $74.2(70.8-77.3)$ & 123,000 & $15.0(12.6-17.8)$ & 54,000 & $6.6(5.0-8.8)$ \\
\hline Wyoming & 51,000 & $70.0(65.8-73.8)$ & 8,000 & $11.4(9.0-14.4)$ & 5,000 & $7.3(5.3-10.2)$ \\
\hline
\end{tabular}

See table footnotes on the next page.

findings could help health care providers encourage patients to participate in these common activities, including referring them to low-cost physical activity programs delivered by worksites and community organizations.

The cost of physical activity is an important consideration for adults with arthritis (7). Whereas all adults with arthritis can benefit from physical activity, those with the lowest levels of household income are more likely to be inactive (8). In this report of adults who engaged in physical activity, type of physical activity varied by income level. For example, adults with lower socioeconomic status had lower weightlifting and higher walking prevalences compared with those with higher 
TABLE 3. (Continued) Unadjusted reported prevalence of walking, gardening, or weightlifting as a first or second most frequent activity* among adults with arthritis ${ }^{\dagger}$ who reported engaging in physical activity in the past month ${ }^{\S}$ - Behavioral Risk Factor Surveillance System, United States, 2019

\begin{tabular}{|c|c|c|c|c|c|c|}
\hline \multirow[b]{2}{*}{ Jurisdiction } & \multicolumn{2}{|c|}{ Walking } & \multicolumn{2}{|c|}{ Gardening } & \multicolumn{2}{|c|}{ Weightlifting } \\
\hline & Weighted no.** & $\begin{array}{l}\text { Unadjusted } \\
\%(95 \% \mathrm{Cl})\end{array}$ & Weighted no.** & $\begin{array}{l}\text { Unadjusted } \\
\%(95 \% \mathrm{Cl})\end{array}$ & Weighted no.** & $\begin{array}{l}\text { Unadjusted } \\
\%(95 \% \mathrm{Cl})\end{array}$ \\
\hline $\begin{array}{r}\text { Median (49 states and } \\
\text { District of Columbia) }\end{array}$ & - & 70.5 & - & 12.6 & - & 7.1 \\
\hline Guam & 6,000 & $57.8(47.2-67.8)$ & 2,000 & $25.2(15.5-38.3)$ & 1,000 & 11.9 (7.4-18.8) \\
\hline Puerto Rico & 153,000 & $68.3(63.5-72.8)$ & 22,000 & $9.8(7.3-13.0)$ & 3,000 & $-^{\dagger \dagger}$ \\
\hline
\end{tabular}

Abbreviation: $\mathrm{Cl}=$ confidence interval.

* Adults engaging in physical activity were classified as participating in an activity if they reported this activity for one of two questions: 1) "What type of physical activity or exercise did you spend the most time doing during the past month?" or 2) "What other type of physical activity gave you the next most exercise during the past month?" Participants who reported one activity but had missing data for the other most frequent activity (e.g., "don't know," or "refused") were included in the analysis.

† Respondents were classified as having arthritis if they responded "yes" to the question, "Have you ever been told by a doctor or other health care provider that you have arthritis, rheumatoid arthritis, gout, lupus, or fibromyalgia?"

$\S$ Respondents with arthritis were classified as engaging in physical activity if they responded "yes" to the question, "During the past month, other than your regular job, did you participate in any physical activities or exercises such as running, calisthenics, golf, gardening, or walking for exercise?"

9 In 2019, New Jersey did not collect enough data to meet the minimum requirement for inclusion in the BRFSS public-use data set.

** Weighted number represents the estimated number of adults with arthritis engaging in physical activity who reported the activity (walking, gardening, or weightlifting) as their first or second most frequent activity.

${ }^{\dagger+}$ Unreliable estimate (relative standard error $>30 \%$ ).

incomes. Adults with arthritis who are inactive and have lower incomes might be more receptive to low-cost physical activities, such as walking $(7) .{ }^{\dagger \dagger \dagger}$

Adults with arthritis experience optimal health benefits through diverse physical activity regimens, including aerobic, muscle strengthening, and balance exercises (1). Benefits of gardening include reduced stress and fatigue and improved mental health and quality of life (9). Muscle strengthening can improve fitness and independence, prevent muscle loss, and reduce arthritis pain (1). Low-cost muscle strengthening activity options, including lifting objects (e.g., dumbbells, cans of food, or water bottles), using resistance bands, and engaging in bodyweight exercises, are all suitable activities for adults with arthritis. $\$ \$ \$ \$$

The findings in this report are subject to at least six limitations. First, BRFSS data are self-reported, which can introduce recall and social desirability biases and potential misclassification of activities. Second, the relatively low state-specific response rates (as low as $37.3 \%$ ) might reduce generalizability and bias the findings. Third, specific activity participation might be underestimated because only the two most frequent activities per person could be reported and data were assessed only for leisure-time (nonwork) activities. Fourth, differences in other activities by characteristics such as income were not assessed. Fifth, data was available for only 49 states and aggregated data might not be nationally representative. Finally, this study provides estimates of reported activities undertaken versus preferred; health care providers might find that this affects physical activity sustainability among patients.

\footnotetext{
$\overline{\dagger \dagger \dagger}$ https://www.cdc.gov/arthritis/basics/physical-activity-overview.html

$\$ \mathbb{S}$ https://www.arthritis.org; https://www.cdc.gov/arthritis/interventions/ physical-activity.html
}

To promote physical activity among adults with arthritis, health care providers can offer advice or counseling for walking or referrals to low-cost, evidence-based physical activity programs. ${ }^{\$ \Phi \$}$ These programs might help adults with arthritis overcome common barriers to physical activity, including cost, lack of instructions about preventing risk for injury while exercising, and fear of arthritis worsening (7). Communities can address physical environment barriers to walking by providing safe and supportive infrastructures such as sidewalks, benches, and green spaces.999 Promoting engagement in physical activity among adults with arthritis can reduce their risk for chronic health conditions and improve their mental health and quality of life.

\footnotetext{
999 https://www.thecommunityguide.org/findings/physical-activitybuilt-environment-approaches
}

\section{Acknowledgement}

Oak Ridge Institute for Science and Education.

Corresponding authors: Dana Guglielmo, danagugliel@gmail.com; Janet B. Croft, jbc0@cdc.gov.

\footnotetext{
${ }^{1}$ Division of Population Health, National Center for Chronic Disease Prevention and Health Promotion, CDC; ${ }^{2}$ Oak Ridge Institute for Science and Education, Oak Ridge, Tennessee; ${ }^{3}$ Division of Nutrition, Physical Activity, and Obesity, CDC; ${ }^{4}$ Epidemic Intelligence Service, CDC, National Center for Chronic Disease Prevention and Health Promotion, CDC.
}

All authors have completed and submitted the International Committee of Medical Journal Editors form for disclosure of potential conflicts of interest. No potential conflicts of interest were disclosed. 


\section{Summary}

What is already known about this topic?

Among adults with arthritis, physical activity can reduce pain, disability, and functional limitations, and improve mental health and quality of life; however, just over one third of adults with arthritis are aerobically active.

What is added by this report?

Approximately $71 \%$ of adults with arthritis who engaged in physical activity in the past month reported walking as one of their two most frequent activities. Gardening (13.3\%) and weightlifting $(7.3 \%)$ were the second and third most common activities.

What are the implications for public health practice?

Health care providers can help inactive adults with arthritis become active and, by encouraging physical activity and referring them to evidence-based physical activity programs, improve their health and quality of life.

\section{References}

1. 2018 Physical Activity Guidelines Advisory Committee. 2018 Physical Activity Guidelines Advisory Committee scientific report. Washington, DC: U.S. Department of Health and Human Services, 2018. https:// health.gov/sites/default/files/2019-09/PAG_Advisory_Committee_ Report.pdf
2. Murphy LB, Hootman JM, Boring MA, et al. Leisure time physical activity among US adults with arthritis, 2008-2015. Am J Prev Med 2017;53:345-54. PMID:28601405 https://doi.org/10.1016/j. amepre.2017.03.017

3. Hollis ND, Zhang QC, Cyrus AC, Courtney-Long E, Watson K, Carroll DD. Physical activity types among US adults with mobility disability, Behavioral Risk Factor Surveillance System, 2017. Disabil Health J 2020;13:100888. PMID:32061542 https://doi.org/10.1016/j. dhjo.2020.100888

4. Theis KA, Steinweg A, Helmick CG, Courtney-Long E, Bolen JA, Lee R. Which one? What kind? How many? Types, causes, and prevalence of disability among US adults. Disabil Health J 2019;12:411-21. PMID:31000498 https://doi.org/10.1016/j.dhjo.2019.03.001

5. Hootman JM, Theis KA, Barbour KE, Paul P, Carlson SA. Leisure time and transportation walking among adults with and without arthritis in the United States, 2010. Arthritis Care Res (Hoboken) 2019;71:178-88. PMID:30346654 https://doi.org/10.1002/acr.23790

6. Kolasinski SL, Neogi T, Hochberg MC, et al. 2019 American College of Rheumatology/Arthritis Foundation guideline for the management of osteoarthritis of the hand, hip, and knee. Arthritis Care Res (Hoboken) 2020;72:149-62. PMID:31908149 https://doi.org/10.1002/acr.24131

7. Wilcox S, Der Ananian C, Abbott J, et al. Perceived exercise barriers, enablers, and benefits among exercising and nonexercising adults with arthritis: results from a qualitative study. Arthritis Rheum 2006;55:616-27. PMID:16874785 https://doi.org/10.1002/art.22098

8. Guglielmo D, Murphy LB, Boring MA, et al. State-specific severe joint pain and physical inactivity among adults with arthritis-United States, 2017. MMWR Morb Mortal Wkly Rep 2019;68:381-7. PMID:31048678 https://doi.org/10.15585/mmwr.mm6817a2

9. Soga M, Gaston KJ, Yamaura Y. Gardening is beneficial for health: a meta-analysis. Prev Med Rep 2017;5:92-9. PMID:27981022 https://doi. org/10.1016/j.pmedr.2016.11.007 